\title{
THE BASIC PROPERTIES OF BLOCH FUNCTIONS
}

\author{
JOSEPH A. CIMA \\ Mathematics Department \\ The University of North Carolina \\ at Chapel Hill \\ Chapel Hill, North Carolina 27514 \\ U.S.A. \\ (Received February 2, 1979)
}

ABSTRACT. A Bloch function $\mathrm{f}(\mathrm{z})$ is an analytic function on the unit disc $D$ whose derivative grows no faster than a constant times the reciprocal of the distance from $z$ to $\partial \mathbf{D}$. We reprove here the basic analytic facts concerning Bloch functions. We establish the Banach space structure and collect facts concerning the geometry of the space. We indicate duality relationships, and known isomorphic correspondences are given. We give a rather complete list of references for further study in the case of several variables.

KEY WORDS AND PHRASES. Bloch function, Schlicht discs, Normal functions, isomorphic Banach spaces, Mobius invariant subspaces.

1980 MATHEMATICS SUBJECT CLASSIFICATION CODES. 30A78, $46 E 15$. 
1. Introduction.

The purpose of this article is to give a survey and some proofs of known results concerning Bloch functions. The basic idea goes back to Andre Bloch [ 6 ]. He considered the class $F$ of functions holomorphic on the unit disc $\mathbb{D}$, with normalization $f^{\prime}(0)=1$. The image of $D$ under $f$ is considered as a Riemann surface $w_{f}=f(\mathbb{D})$. A schlicht (unramified) disc in $w_{f}$ is an open disc $\Delta \subset w_{f}$ such that there exists a domain $\Omega \subseteq \mathbb{D}$ with $f$ mapping $\Omega$ one to one onto $\Delta$. We denote the radius of the largest schlicht disc in $w_{f}$ with center $f(z)$ as $d_{f}(z)$. Let $r_{f}$ be the supremum of $d_{f}(z)$ as $z$ varies over $\mathbb{D}$ and set

$$
b=\inf \left\{r_{f}: f \in F\right\}
$$

Bloch showed that $b$ was positive.

During the period from 1925 through 1968 Bloch's result motivated works of various nature. One group of mathematicians considered the generalizations of Bloch's result to balls in $\mathbb{R}^{n}$ and $\phi^{n}$. A group of mathematicians calculated upper and lower bounds for b. A third group concentrated on the function theoretic implications for the case of the disc. The Bloch theorem has been an ingredient in supplying a proof of the Picard theorem which avoids the use of the modular function. We will not go into the generalizations for the $\mathrm{n}$ dimensional case but refer the interested reader to the papers of S. Bochner [ 7 ], S. Takahashi [ 25 ] and $\mathrm{K}$. Sakaguchi [ 20 ]. We will also not discuss the best bounds but only refer to the papers of L. V. Ahlfors [ 1 ], L. V. Ahlfors and H. Grunsky [ 2 ] and M.H. Heins [ 13 ]. 
In the period from 1969 to the present a Banach space B of holomorphic functions called the "Bloch functions" has been studied. of course the requirement for membership in $B$ is derived from the idea of the Bloch theorem. Some progress has been made in studying the functional analytic properties of $B$. The Banach space point of view has allowed a somewhat broader viewpoint and consequently has given rise to a new set of questions concerning the Bloch space.

This article will give a proof of the basic Bloch theorem. We will follow a theme developed by W. Seidel and J. Walsh [24] and by Ch. Pommerenke [17]. We will supply proofs of the major results and outline proofs of other ideas when they are not central to our interests. We have borrowed freely from the text material available (especially M. Heins [13]). In many instances we have selected only partial results from the journal articles quoted in the bibliography. The reader should consult the original article if he desires a more complete exposition. Finally, I wish to point out a few other results which will not be included in this article but are extremely important to the overall picture concerning Bloch functions. First, L. A. Harris [12] has obtained a strong form of the Bloch theorem for holomorphic mappings from the unit ball $B$, of a Banach space $X$ into $X$. The second topic concerns the thesis of R. Timoney. He has made a definitive study of Bloch functions on bounded symmetric domains in $\phi^{n}$. This work is quite expansive and deep and would require material from areas which are not considered in the disc case.

2. The Theorem of Bloch.

Let Aut (D) denote the group of holomorphic automorphisms of $\mathbb{D}$. For $a \in \mathbb{D}$ we write $\phi_{a}(z)=(z-a)(1-\bar{a} z)^{-1} \in$ Aut (D). The inverse of 
$\phi_{a}$ is $\phi_{-a}$. It can be shown that for $z \in \mathbb{D}$,

$$
\left|\phi_{|a|}(|z|)\right| \leq\left|\phi_{-a}(z)\right|
$$

Let $a \in(0,1)$ and denote $A_{a}=A$ the family of holomorphic functions $f: D \rightarrow D$ with normalization $f(0)=0, f^{\prime}(0)=a$. A is a nonempty compact family. It contains the function

$$
B(z)=-\phi_{0}(z) \cdot \phi_{a}(z) .
$$

For $f \in A$ set

$$
\begin{gathered}
U_{f} \equiv \sup \left\{r \mid f \quad \text { is univalent in } D_{r}=(|z|<r)\right\} \\
\text { and } \\
\rho \equiv \inf \left\{U_{f}: f \in A\right\}
\end{gathered}
$$

A calculation shows that $B^{\prime}$ vanishes at a point of $\mathbb{D}$, hence $\rho<1$.

Theorem (2.1). The number $\rho$ is positive. The number $u_{f}=\rho$ if and only if

$$
f(z)=\bar{\lambda}_{B}(\lambda)
$$

where $\lambda$ is a constant of modulus one.

Proof. Fix $f \in A$ and assume $U_{f}<1$. Recalling the normalization of $f$ we see that equation

$$
\phi_{a}\left(\frac{f(z)}{z}\right)=z k(z)
$$

yields a function $k$, holomorphic in $D$ and bounded by one. Hence, by (2.1) 


$$
\begin{aligned}
& |f(z)|=\left|z \phi_{-a}(z k(z))\right| \\
& \geq|z|\left(\frac{a-|z k(z)|}{1-a \mid z k(z)}|\geq| z \mid\left(\frac{a-|z|}{1-a|z|}\right) \equiv \psi(|z|) .\right.
\end{aligned}
$$

E1ther $f$ fails to be one to one on $|z| \leq u_{f}$ or there exists a $z_{0},\left|z_{0}\right|=u_{f}$ and $f^{\prime}\left(z_{0}\right)=0$. We will complete the proof by assuming that there exists distinct points $z_{1}$ and $z_{2},\left|z_{1}\right|=\left|z_{2}\right|=u_{f}$ and $f\left(z_{1}\right)=f\left(z_{2}\right)=c$. The other case is similar. The function

$$
m(z)=\left(\phi_{c} \circ f(z)\right) \cdot \phi_{z_{1}}^{-1}(z) \cdot \phi_{z_{2}}^{-1}(z)
$$

has removable singularities and is bounded by one in $\mathbb{D}$. Hence, $|m(0)| \leq \mid$ implying $|c| \leq u_{f}^{2}$. Setting $z=z_{1}$ in (2.1) and using the last inequality we have

$$
u_{f} \geq\left(\frac{a-u_{f}}{1-a u_{f}}\right) \text {. }
$$

The functions $x$ and $-\phi_{a}(x)$ have a common value at $x_{0}=a^{-1}\left(1-\sqrt{1-a^{2}}\right) \in(0,1)$ and $-\phi_{a}(z) \geq x$ if $0<x<x_{0}$. Thus $u_{f} \geq a^{-1}\left(1-\sqrt{1-a^{2}}\right)$. We note that $B^{\prime}\left(x_{0}\right)=0$. Thus $\rho=u_{B}$. The remaining uniqueness part of the theorem is handled by noting that if $u_{f}=\rho$ then $|m(0)|=1$.

We proceed to a second necessary result. For $r>0$ let $D_{\mathbf{r}}=\{|w|<\mathbf{r}\}$. To each $\mathbf{f} \in \mathrm{A}$ let

$$
\ell_{f}=\sup \left\{r \mid \exists \text { a domain } \Omega_{r} \subseteq D\right. \text { such }
$$

that $0 \in \Omega_{r}$ and $f$ maps $\Omega_{r}$ univalently onto $\left.D_{r}\right\}$

$$
s=\inf \left\{l_{f}: \mathbf{f} \in A\right\} .
$$


Theorem 2.2. The number $s$ is positive, equaling

$$
\rho^{2}=\left[a^{-1}\left(1-\sqrt{1-a^{2}}\right)\right]^{2} .
$$

Equality holds for some $f$ in $A, \ell_{f}=s$, if and only if $f(z)=\bar{\lambda} B(\lambda z)$ for same constant $\lambda,|\lambda|=1$.

Proof: For any $f \in A$ inequality (2.2) implies that for $|z|=r<1$

$$
|f(z)| \geq \min _{|z|=r}|f(z)| \geq \psi(r)=r\left(\frac{a-r}{1-a r}\right) .
$$

$\psi$ is a positive function on $(0, a)$ and achieves a maximum value of $\rho^{2}$ at $r=\rho(<a)$. Let $\Omega$ be the component of $f^{-1}\left(\mathbb{D}_{\rho^{2}}\right)$ containing zero. If there is $z \in\left(\mathbb{D} \backslash \mathbb{D}_{\rho}\right) \cap \Omega$ then there exists a $z^{*},\left|z^{*}\right|=\rho$ and $f\left(z^{*}\right) \in \mathbb{D}_{\rho^{2}}$. By inequality (2.3) this is absurd. Hence $\Omega \subseteq \mathbb{D}_{\rho}$. Using (2.3) again and the open mapping property one sees that $f$ maps the boundary of $\Omega$ into $\left(|w|=\rho^{2}\right)$ and maps $\Omega$ onto $D_{\rho}$. Since $f(z)=0$ has only one solution in $\Omega$ it follows by the argument principle that $f$ maps the (simp1y connected) domain $\Omega$ univalent1y onto $\mathbb{D}_{\rho} 2$. By definition it follows that $\rho^{2} \leq s$. Since $\psi(\rho)=B(\rho)=\rho^{2}$ and $B^{\prime}(\rho)=0$ we observe that $B$ cannot be univalent on any domain containing $\rho$. Thus $\rho^{2} \leq s \leq \ell_{B} \leq \rho^{2}$ and so $s=\rho^{2}$. The remaining uniqueness results follow in a routine way by a close examination of when equality holds in (2.2)

We are finally in a position to prove Bloch's theorem. The notation is that used in the introduction.

Theorem (2.3). The number $b$ is positive. 
Proof. Elementary considerations show that for $f \in F, 0<t<1$ and $g(z)=f(t z) t^{-1}$ we have $r_{g} \leq r_{f} \cdot t^{-1}$. Thus it is sufficient to consider those $f \in F$ which are analytic on the closed disc $\bar{D}$. Let $f$ be so chosen and let $|a|<1$ be selected so that

$$
\max _{\mathbf{z} \in \mathbf{D}}\left|\mathrm{f}^{\prime}(\mathrm{z})\right|\left(1-|z|^{2}\right)=\left|\mathrm{f}^{\prime}(\mathrm{a})\right|\left(1-|\mathbf{a}|^{2}\right) \geq 1
$$

Now form the function

$$
g(z)=\frac{f \circ \phi_{-a}(z)}{\left(f \circ \phi_{-a}\right)^{\prime}(0)} .
$$

Note that $g \in F$ and is analytic on $\bar{D}$ with

$$
r_{g} \leq \frac{1}{\mid\left(f \circ \phi_{-a}\right)^{\prime}(0)} \mid r_{f} \leq r_{f}
$$

Further,

$$
\left|f^{\prime}\left(\frac{a+z}{1+\bar{a} z}\right)\right|\left(1-\left|\frac{z+a}{1+z}\right|^{2}\right) \leq\left|f^{\prime}(a)\right|\left(1-|a|^{2}\right)
$$

and so

$$
\begin{gathered}
\left(1-|z|^{2}\right)\left|g^{\prime}(z)\right| \leq 1 . \\
\text { Replacing } g \text { by } h(z)=g(z)-g(0) \text { and integrating we deduce } \\
|h(z)| \leq \frac{1}{2} \log \frac{1+|z|}{1-|z|} \equiv \frac{1}{2} L(|z|) .
\end{gathered}
$$

Let $t$ be fixed in $(0,1)$ and normalize $h$ as follows

$$
S_{t}(z)=\frac{h(t z)}{\frac{1}{2} L(t)} .
$$

$S_{t}$ maps $\mathbb{D} \rightarrow \mathbb{D}, \quad S(0)=0$ and $S_{t}^{\prime}(0)=2 t / L(t)$. The number $\frac{2 t}{L(t)}=a$ is in $(0,1)$. Hence, $s_{t} \in A_{a}$. We apply Theorem (2.2) to $s_{t}$ and note 


$$
r_{S_{t}} \geq\left[\frac{L(t)}{2 t}\left(1-\sqrt{1-\left(\frac{2 t}{L(t)}\right)^{2}}\right)\right]^{2}
$$

Since, $r_{f} \geq r_{h_{t}}$ we conclude

$$
r_{f} \geq \frac{1}{2} L(t)\left[\left(\frac{2 t}{L(t)}\right)\left(1+\sqrt{1-\left(\frac{2 t}{L(t)}\right)^{2}}\right)^{-1}\right]^{2} .
$$

If one checks the value of this expression (preferably with a calculator) at $t=\frac{1}{2}$ one concludes $r_{f} \geq 0.2$.

The papers refered to in the introduction establish the bounds

$$
\frac{1}{4} \sqrt{3}<b<0.472
$$

We proceed to the paper of W. Seidel and J. Walsh [24]. They introduced the expression $d_{f}(z)=d(z)$ as the radius of the largest schlicht disc in $f(D)$ with center $f(z)$. In this paper they collect some known results concerning $d_{f}(z)$ and they prove several theorems about its growth. The following two theorems are interesting and motivating.

Theorem (2.4). Let $f$ be holomorphic and univalent in $\mathbb{D}$. Then for $z \in \mathbb{D}$

$$
d(z) \leq\left|f^{\prime}(z)\right|\left(1-|z|^{2}\right) \leq 4 d(z) .
$$

Proof. Let $z_{0} \in D$ be given. We prove first the right inequality. Form the function

$$
\psi(z)=\frac{f \circ \phi_{-z_{0}}(z)-f\left(z_{0}\right)}{f^{\prime}\left(z_{0}\right)\left(1-\left|z_{0}\right|^{2}\right)}
$$

where $z \in \mathbb{D} . \quad \psi$ is a normalized univalent function and if $\omega \notin f(\mathbb{D})$ then $\psi$ omits the value $\alpha$, 


$$
\alpha=\frac{\omega-f\left(z_{0}\right)}{\left(f^{\prime}\left(z_{0}\right)\right)\left(1-\left|z_{0}\right|^{2}\right)} .
$$

Applying the Koebe one-quarter theorem and choosing $\omega$ suitably on $\partial f(D)$ we conclude that

$$
\frac{d\left(z_{0}\right)}{\left(1-\left|z_{0}\right|^{2}\right) f^{\prime}\left(z_{0}\right)} \geq \frac{1}{4} .
$$

To obtain the left inequality again let $z_{0}$ be fixed in $\mathbb{D}$ and form a function $\psi(z)=f \circ \phi_{-z_{0}}(z)$. If $f\left(z_{0}\right)=\omega_{0}(=\psi(0))$ then $\psi$ has an inverse $h$ mapping the disc $\left|\omega_{0}-\omega\right|<d\left(z_{0}\right)$ into 0 with $h\left(\omega_{0}\right)=0$. The Schwarz lemma yields $\left|h^{\prime}\left(\omega_{0}\right)\right| \leq\left(d\left(z_{0}\right)\right)^{-1}$ and this completes the proof.

Notice that the univalence of $f$ is not used in the proof of the left inequality and hence $d_{f}(z) \leq\left|f^{\prime}(z)\right|\left(1-|z|^{2}\right)$ is valid for any holomorphic f. A second result of interest in that paper is the following. Theorem (2.5). Let $f$ be a bounded holomorphic function on $\mathbb{D}$, $|f(z)| \leq \| f||_{\infty}=M$. Then

$$
d(z) \leq\left|f^{\prime}(z)\right|\left(1-|z|^{2}\right) \leq[8 M d(z)]^{\frac{1}{2}} .
$$

Outline of proof. We have noted that the left inequality is valid. The idea of the proof in the right inequality is to $f i x z_{0} \in \mathbb{D}$ and form the function

$$
\psi(z)=\frac{f \circ \phi_{-z_{0}}(z)-f\left(z_{0}\right)}{f^{\prime}\left(z_{0}\right)\left(1-\left|z_{0}\right|^{2}\right)}=z+\ldots .
$$

Then $\psi(z)=0$ and $|\psi(z)| \leq \frac{2 M}{\left|f^{\prime}\left(z_{0}\right)\right|\left(1-\left|z_{0}\right|^{2}\right)}$. 
One can then normalize $\psi$ and apply a variant of Theorem (2.2) to conclude that $\psi$ covers the disc

$$
|\xi| \leq \frac{\left|f^{\prime}\left(z_{0}\right)\right|\left(1-\left|z_{0}\right|^{2}\right)}{8 M}
$$

univalently. This in turn implies that $f$ covers the disc

$$
\left|\omega-f\left(z_{0}\right)\right| \leq \frac{\left|f^{\prime}\left(z_{0}\right)\right|^{2}\left(1-\left|z_{0}\right|^{2}\right)}{8 M}
$$

univalently. From this it follows that

$$
\left|f^{\prime}\left(z_{0}\right)\right|\left(1-\left|z_{0}\right|^{2}\right) \leq\left[8 \mathrm{Md}\left(z_{0}\right)\right]^{\frac{1}{2}} \text {. }
$$

The next theorem is a statement of several equivalences. The term Bloch function will be defined by any of these equivalent conditions. The equivalence of (1) and (2) is in Seidel and Walsh [ 24 ]. The fifth condition is due to Zygmund. The last is in Anderson and Rubel [ 4 ]. The conditions (3) and (4) were given by Pommerenke [ 17 ]. We recall a few terms. A holomorphic function $f(z)$ (on $D$ ) is said to be finitely normal if the family of functions

$$
\begin{array}{r}
\left\{f_{\phi}(z) \equiv f(\phi(z))-f(\phi(0)) ;\right. \\
\phi \in \text { Aut (D) }\}
\end{array}
$$

is a normal family, where the constant infinity is not allowed as a limit. A function $g$ continuous on $|z|=1$ is in the class $\Lambda_{\star}$ if the following condition holds

$$
\left|g\left(e^{i(\theta+h)}\right)-2 g\left(e^{i \theta}\right)+g\left(e^{i(\theta-h)}\right)\right|=O(h)
$$


uniformly for all $\theta \in[0,2 \pi)$ and $h>0$.

For $f$ holomorphic in $D$, the absolute convex hull of the orbit of $f$ under Aut (D) is defined as the family

$$
\begin{aligned}
& \left\{\sum_{j=1}^{n} a_{j}\left(f \circ \phi_{j}\right)(z): n \in N, a_{j} \in \operatorname{Aut}(D),\right. \\
& \left.a_{j} \in \phi \text { with } \sum_{i}^{n}\left|a_{j}\right| \leq \mid\right\} .
\end{aligned}
$$

Theorem (2.6). The following conditions on a function $f$ holomorphic in $D$ are equivalent:

(1) $\sup \{d(z): z \in \mathbb{D}\}<+\infty$.

(2) $\sup \left\{\left(1-|z|^{2}\right)\left|f^{\prime}(z)\right|: z \in \mathbb{D}\right\}<+\infty$.

(3) $f$ is finitely normal.

(4) there exists a constant $\alpha>0$ and a univalent holomorphic $g$ on D such that

$$
f(z)=\alpha \log g^{\prime}(z) .
$$

(5) the primitive

$$
g(z) \equiv \int_{0}^{z} f(t) d t
$$

is in the disc algebra and $g\left(e^{i \theta}\right) \in \Lambda_{*}$.

(6) The absolute convex hull of $f$ is a normal family on $\mathbb{D}$.

Proof: We know that (2) implies (1). Assume then $d(z) \leq M$ for $z \in \mathbb{D}$. Form the functions $\psi$ and $g$

$$
\psi(z)=f \circ \phi_{-z_{0}}(z) \quad \text { and }
$$


380 J. A. CIMA

$$
g(z)=\frac{\psi\left[z_{1}+\left(1-\left|z_{1}\right|\right) z\right]}{\left(1-\left|z_{1}\right|\right) \psi^{\prime}\left(z_{1}\right)}
$$

where we have $z_{0}, z_{1} \in \mathbb{D}$ and for the moment we assume $\psi^{\prime}\left(z_{1}\right) \neq 0$. The function $g$ is analytic with $g^{\prime}(0)=1$. It follows by Bloch's theorem that there exists $\zeta * \in \mathbb{D}$ such that $\mathrm{dg}\left(\zeta^{*}\right) \geq \mathrm{b}$. Then with $\omega *=z_{1}+\left(1-\left|z_{1}\right|\right) \zeta *$

$$
\mathrm{d} \psi\left(\omega^{*}\right) \geq \mathrm{b} \quad\left(1-\left|\mathrm{z}_{1}\right|\right)\left|\psi^{\prime}\left(\mathrm{z}_{1}\right)\right|
$$

Note that if $\psi^{\prime}\left(z_{1}\right)=0$ the above inequality is still valid. Hence, for all $z \in \mathbb{D}$ we have

$$
\left|\psi^{\prime}(z)\right|(1-|z|) \leq M / b .
$$

Normalize $\psi$ by considering

$$
\Phi(z)=\psi(z)-\psi(0)=\int_{0}^{z} \psi^{\prime}(t) d t .
$$

Fixing $t \in(0,1)$ and noting that

$$
|\Phi(z)| \leq-\frac{M}{b} \log (1-t)
$$

if $|z| \leq t$, we apply Theorem (2.5) to the function $\eta(z) \equiv \Phi(t z)$ to conclude

$$
t^{2}\left|f^{\prime}\left(z_{0}\right)\right|^{2}\left(1-\left|z_{0}\right|^{2}\right) \leq \frac{8 M}{b}(-\log (1-t)) d_{f}\left(z_{0}\right)
$$

The maximum of the function $U(t)=\left(-t^{2}\right)(\log (1-t))^{-1}$ occurs when $-\log (1-t)=t / 2(1-t) \quad(0<t<1)$ which is approximately $t_{0} \simeq 072$. Hence,

$$
d_{f}\left(z_{0}\right) \geq \frac{b}{8 M} U\left(t_{0}\right)\left|f^{\prime}\left(z_{0}\right)\right|^{2}\left(1-\left|z_{0}\right|^{2}\right)^{2}
$$

Assume that (2) holds and recall that a family $\left\{\mathrm{f}_{\phi}\right\}$ defined and 
holomorphic on $\mathbb{D}$ is normal if for every compact $K \subset \mathbb{D}$ there exists a positive number $M(K)$ such that

$$
\sup _{z_{\epsilon} K}\left(\frac{\left|f_{\phi}^{\prime}(z)\right|}{1+\left|f_{\phi}(z)\right|^{2}}\right) \leq M(K) .
$$

Thus for $\phi \in$ Aut (D) and $K \subset D$ compact

$$
\begin{aligned}
& \sup _{z \in K}\left(\frac{\left|f_{\phi}^{\prime}(z)\right|}{\left(1+\left|f_{\phi}(z)\right|^{2}\right)}\right)=\sup _{z \in K}\left(\frac{\left|f^{\prime}(\phi(z))\right| \mid\left(\phi^{\prime}(z) \mid\right.}{\left(1+\left|f_{\phi}(z)\right|^{2}\right)}\right) \\
& =\sup _{z \in K} \frac{1}{\left(1-|z|^{2}\right)}\left(\frac{\left(1-|\phi(z)|^{2}\right)\left|f^{\prime}(\phi(z))\right|}{\left(1+\left|f_{\phi}(z)\right|^{2}\right)}\right)<\infty \text {. }
\end{aligned}
$$

For the converse let $w \in \mathbb{D}$ and choose $\psi(z)=(z+w)(1+\bar{w} z)^{-1}$. There is a positive number $M(0)$ such that

$$
\frac{\left|f_{\phi}^{\prime}(0)\right|}{1+\left|f_{\phi}(0)\right|^{2}} \leq M(0)
$$

for all $\phi \in \operatorname{Aut}(D)$. Thus

$$
\left(1-|w|^{2}\right)\left|f^{\prime}(w)\right|=\left|\psi^{\prime}(0)\right|\left|f^{\prime}(\psi(0))\right| \leq M(0) \text {. }
$$

The equivalence of (2) and (4) is dependent upon some results from the theory of univalent functions. Let $f(z)$ have the representation $\alpha \log g^{\prime}(z)$, where $g$ is univalent and $\alpha>0$. It is known [ 11$]$ that for such $g$

$$
\left|\frac{g^{\prime \prime}(z)}{g^{\prime}(z)}\right| \leq \frac{6}{1-|z|^{2}} \quad, z \in D \text {. }
$$

The expression $\left(1-|z|^{2}\right)\left|f^{\prime}(z)\right|$ is then bounded by $6 \alpha$. If (2) holds define $\alpha>0$ by the equality 


$$
\alpha^{-1} \equiv 3 \sup \left\{\left(1-|z|^{2}\right)\left|f^{\prime}(z)\right|: z \in \mathbb{D}\right\}
$$

and define $g$ so that

$$
g(z)=\int_{0}^{z} \exp \left[\frac{f(\zeta)}{\alpha}\right] d \zeta .
$$

The definitions of $g$ and $\alpha$ yields

$$
\left(1-|z|^{2}\right)\left|\frac{g^{\prime \prime}(z)}{g^{\prime}(z)}\right| \leq \frac{1}{3}
$$

This is sufficient to conclude that $g$ is univalent.

The proof that (2) and (5) are equivalent requires a lemma.

Lemma (2.7). Let $f$ be analytic in $\mathbb{D}$ with $f^{\prime \prime}(z)=O\left((1-|z|)^{-1}\right)$, then $f$ is continuous on $\bar{D}$.

Proof. The growth condition on $f^{\prime \prime}$ implies that $f^{\prime}(z)=O(\log (1-|z|))$. An integration of $f^{\prime}$ proves that $f$ is a bounded holomorphic function and has radial limits at each point of $e^{i \theta} \epsilon \partial D$. If $\theta_{1}, \theta_{2} \in[0,2 \pi)$ and $0<p<1$

$$
\begin{aligned}
& \Gamma_{j}=\left\{r e^{i \theta}: \rho \leq r<1\right\} \quad j=1,2 \\
& \Gamma_{3}=\left\{\rho e^{i \theta}: \theta_{1} \leq \theta \leq \theta_{2}\right. \\
& \Gamma_{4}=\left\{e^{i \theta}: \theta_{1} \leq \theta \leq \theta_{2}\right\}
\end{aligned}
$$

the Cauchy integral theorem implies

$$
\begin{aligned}
f\left(e^{i \theta}\right)-f\left(e^{i \theta} 1\right)= & \frac{1}{\partial \Pi i} \int f^{\prime}(\delta) d \delta . \\
& -\Gamma_{1} \cup \Gamma_{2} \cup \Gamma_{3}
\end{aligned}
$$

Each of the integrals can be evaluated and there is a constant $c>0$ 
(independent of $\theta_{i}$ ) such that

$$
\left|f\left(e^{i \theta} 2\right)-f\left(e^{i \theta} 1\right)\right| \leq c\left|\theta_{2}-\theta_{1}\right||\log | \theta_{2}-\theta_{1}|| .
$$

This completes the lemma.

Proof of the equivalence of (2) and (5). Assume (2) holds. Lemma (2.7) implies that $f$ is continuous on $\bar{D}$. Let $1>h>0$ be given and fixed. It is convenient to assign a symbol to the difference expression

$$
f\left(e^{i(\theta+h)}\right)-f\left(e^{i \theta}\right) \equiv A f(\theta)
$$

and to ignore the dependence of $A$ on $h . A$ is linear and homogeneous. We are required to show

$$
A^{2} f\left(e^{i \theta}\right)=O(h)
$$

uniformly in $\theta,($ as $h \rightarrow 0)$. Fix $t=1-h$ and write

$$
f\left(e^{i \theta}\right)=f\left(e^{i \theta}\right)-f\left(t e^{i \theta}\right)+f\left(t e^{i \theta}\right)
$$

An integration by parts yields that

$$
\begin{gathered}
e^{\partial i \theta} \int_{t}^{1}(1-r) f^{\prime \prime}\left(r e^{i \theta}\right) d r= \\
(1-t) e^{i \theta} f^{\prime}\left(t e^{i \theta}\right)+f\left(e^{i \theta}\right)-f\left(t e^{i \theta}\right)
\end{gathered}
$$

The integral in this equality is $O(h)$, uniformly in $\theta$. With

$$
\begin{gathered}
g(t, \theta) \equiv A\left(e^{i \theta} f^{\prime}\left(t e^{i \theta}\right)\right)= \\
f^{\prime}\left(t e^{i(\theta+h)}\right) A\left(e^{i \theta}\right)+e^{i \theta} A\left(f^{\prime}\left(t e^{i \theta}\right)\right) \\
=O(h|\log h|)+e^{i \theta} A\left(f^{\prime}\left(t e^{i \theta}\right)\right)
\end{gathered}
$$


we can compute

$$
A\left(f^{\prime}\left(t e^{i \theta}\right)=i t \int_{0}^{h} e^{i(\theta+s)} f^{\prime \prime}\left(t e^{i(\theta+s)} d s=0\right. \text { (1). }\right.
$$

Hence, $g(t, \theta)=0(1)$ and

$$
A^{2} f\left(e^{i \theta}\right)=h A(g(t, \theta))+O(h)=O(h) .
$$

We need only show that

$$
A^{2} f\left(t e^{i \theta}\right)=O(h) .
$$

Since,

$$
\begin{aligned}
& A^{2} f\left(t e^{i(\theta-h)}\right)= \\
& 1 t \int_{0}^{h}\left(e^{i(\theta+s)}-e^{i \theta}\right) f^{\prime}\left(t e^{i(\theta+s)}\right) d s \\
& +1 t \int_{0}^{h} e^{i \theta}\left(f^{\prime}\left(t e^{i(\theta+s)}\right)-f^{\prime}\left(t e^{i(\theta-s)}\right)\right) d s \\
& +1 t \int_{0}^{h}\left(e^{i \theta}-e^{i(\theta-t)}\right) f^{\prime}\left(t e^{i(\theta-s)}\right) d s
\end{aligned}
$$

and the integrands of the first and third integrals are dominated by c $s|\log h|$, while that of the second is dominated by $c s(h)^{-1}$ we conclude that $A^{2} f\left(t e^{i \theta}\right)=O(h)$ uniformly in $\theta$.

Let us show that (5) implies (2). As usual $P(r, \theta)$ is the Poisson kernel. The partials

$$
\begin{aligned}
P_{\theta}(r, \theta) & =-2 r\left(1-r^{2}\right)\left[\sin \theta\left(1-2 r \cos \theta+r^{2}\right)^{-2}\right] \\
P_{\theta \theta}(r, \theta) & =\frac{2 r\left(1-r^{2}\right)\left[2 r\left(1+\sin ^{2} \theta\right)-\cos \theta\left(1+r^{2}\right)\right]}{\left(1-2 r \cos \theta+r^{2}\right)^{3}}
\end{aligned}
$$

have the following properties. The second derivative $P_{\theta \theta}$ is an even function of $\theta$ and 


$$
\int_{0}^{\Pi} P_{\theta \theta}(r, t) d t=0
$$

For $r$ fixed in $(0,1), P_{\theta \theta}$ is zero on $(0, \Pi)$ only at the point $s(\theta)=s$ which satisfies

$$
\frac{\cos s}{1+\sin ^{2} s}=\frac{2 r}{1+r^{2}}
$$

A computation shows that

$$
s \simeq \sqrt{3}(1-r)
$$

Since $f$ is in the disc algebra it has a representation

$$
f(z)=\frac{1}{2 \pi} \int_{-\Pi}^{\Pi} P(r, \theta-t) f\left(e^{i t}\right) d t
$$

where $z=r e^{i \theta}$. Taking the partials and changing variables yields

$$
\begin{aligned}
& f_{\theta \theta}(z)=\frac{1}{2 \pi} \int_{0}^{\Pi} P_{\theta \theta}(r, t)\left\{f\left(e^{i(\theta+t)}\right)+f\left(e^{i(\theta-t)}\right)\right\} d t \\
= & \frac{1}{2 \pi} \int_{0}^{\Pi} P_{\theta \theta}(r, t)\left\{f\left(e^{i(\theta+t)}\right)-2 f\left(e^{i \theta}\right)+f\left(e^{i(\theta-t}\right)\right\} d t .
\end{aligned}
$$

The hypothesis $f \in \Lambda_{\star}$ implies

$$
\left|f_{\theta \theta}(z)\right| \leq A \int_{0}^{\Pi} t\left|P_{\theta \theta}(r, t)\right| d t .
$$

We compute the integral on the right

$$
\begin{aligned}
& -\int_{0}^{s} t P_{\theta \theta}(r, t) d t+\int_{s}^{\Pi} t P_{\theta \theta}(r, t) d t= \\
& -2 s P_{\theta}^{\prime}(r, s)+2 P(r, s)-P(r, 0)-P(r, \pi) .
\end{aligned}
$$

The expression 


$$
-2 s P_{\theta}(r, s) \simeq \frac{4 r(1-r)^{3}}{(1-r)^{4}}
$$

and so there is a constant $c$ so that

$$
0 \leq-2 s P_{\theta}(r, s) \leq c(1-r)^{-1}
$$

A similar inequality is valid for the $P(r, s)$ term. Hence

$$
f_{\theta \theta}(z)=O\left((1-|z|)^{-1}\right) \text {. }
$$

The Poisson integral representation for $f$ implies

$$
\left|f_{\theta}(z)\right| \leq c\left(1-r^{2}\right)|| f||_{\infty} \quad \int_{0}^{\pi} \frac{2 r \sin t d t}{\left(1-2 r \cos t+r^{2}\right)^{2}},
$$

thus

$$
f_{\theta}(z)=O\left((1-|z|)^{-1}\right) \text {. }
$$

An easy computation yields

$$
\begin{gathered}
f^{\prime \prime}(z)=r^{-2} e^{-2 i \theta}\left\{i f_{\theta}(z)-f_{\theta \theta}(z)\right\} \\
=O\left((1-r)^{-1}\right) .
\end{gathered}
$$

This completes the equivalence of (2) with (5). We omit the proof for (6).

A perusal of these equivalences and a slight amount of study give some insight into the size of the set of Bloch functions. We have observed that the bounded holomorphic functions are Bloch functions. In general there are no containment relationships between the classical Hardy spaces ${ }_{\mathrm{H}}^{\mathrm{P}}(\mathrm{D}) \quad(0<\mathrm{p}<\infty)$ and the set of Bloch functions. In fact one can construct Bloch functions using gap series which are not of bounded characteristic. 
Although a BLoch function need not have angular (non-tangential) limits almost everywhere on $\partial \mathbb{D}=\{|z|=1\}$, one can prove using the Gross star theorem (from the theory of cluster sets) that every Bloch function has finite or infinite angular limits on an uncountably dense subset of $2 \mathrm{DD}$. yie1ds

If $f(z)=\sum_{n=0}^{\infty} a_{n} z^{n}$ is a Bloch function a straight forward estimate

$$
\left|a_{n}\right| \leq c \quad \sup \left\{\left(1-|z|^{2}\right)\left|f^{\prime}(z)\right|: z \in D\right\}
$$

for $n=1,2,3, \ldots$. The following result will be useful in posing some unanswered questions later in the paper.

Theorem (2.8). Let $f(z)=\sum_{j=1}^{\infty} a_{j} z^{j}$ be a Bloch function with radial limits almost everywhere on $2 \mathbb{D}$. Then $a_{n} \rightarrow 0$.

Proof. Integrating $\left|f^{\prime}(z)\right|^{2}$

$$
\sum_{j=1}^{\infty} j^{2}\left|a_{j}\right|^{2} r^{2 j-2}=\frac{1}{2 \pi} \int_{0}^{2 \pi}\left|f^{\prime}\left(r e^{i \theta}\right)\right|^{2} d \theta
$$

Choosing $r_{n}=\left(1-\frac{1}{n}\right)$ we can find a constant $c>0$ so that

$$
\frac{1}{n^{2}} \sum_{j=1}^{n} j^{2}\left|a_{j}\right|^{2} \leq \frac{c}{2 \pi} \int_{0}^{2 \pi}\left(1-r_{n}\right)\left|f^{\prime}\left(r_{n} e^{i \theta}\right)\right|^{2} d \theta
$$

A known property of normal functions (see Lehto and Virtanen [ 15 ]) states that if $f$ is analytic in $\mathbb{D}$ with radial limit $A$ at a point $\lambda=e^{\text {i } \theta}$ then $f$ has angular limit $A$ at $\lambda$. With $\lambda=e^{1 \theta}$ assume $\lim f(r \lambda)=A$. The discs $r \rightarrow 1$

$$
c_{n}=\left\{\left|z-r_{n} \lambda\right|<\frac{1}{2}\left(1-r_{n}\right)\right\}
$$

lie in a fixed angle at $\lambda$ and tend to $\lambda$ as $n \rightarrow \infty$. Apply the Cauchy formula 


$$
\begin{gathered}
\left(1-r_{n}\right)\left|f^{\prime}\left(r_{n} \lambda\right)\right| \\
=\left(1-r_{n}\right)\left|\frac{1}{2 \pi 1} \int_{\partial c_{n}} \frac{(f(z)-A)}{\left(z-r_{n} \lambda\right)^{2}} d z\right| \\
\leq 2 \max _{z \in \partial c_{n}}|f(z)-A| \rightarrow \underset{n \rightarrow \infty}{\rightarrow 0} .
\end{gathered}
$$

Since the integrand $\left(1-r_{n}\right)\left|f^{\prime}\left(r_{n} e^{1 \theta}\right)\right|$ is uniformly bounded we may apply the Lebesgue bounded convergence theorem to conclude.

$$
\lim _{n \rightarrow \infty} \frac{1}{n^{2}}\left(\sum_{j=1}^{n} j^{2}\left|a_{j}\right|^{2}\right)=0 .
$$

Thus $a_{n} \rightarrow 0$.

3. The Banach space structure.

Theorem (2.6) implies that the set of Bloch functions $B$ is a complex vector space. It is possible to equip $B$ with a norm in which it becomes a Banach space. If $f \in B$ we can define

$$
\|f\|_{B}=|| f|| \equiv|f(0)|+\sup \left\{\left|f^{\prime}(z)\right|\left(1-|z|^{2}\right): z \in D\right\} .
$$

The addition of the term $|f(0)|$ is to account for the constant functions. We prefer to work with the quantity $\sup \left\{\left|f^{\prime}(z)\right|\left(1-|z|^{2}\right)\right\}=M(f)$ and so limit ourselves to functions holomorphic on $D$ for which $f(0)=0$ and

$$
\left.|| f\right|_{B}=M(f)
$$

Henceforth when norm considerations for Bloch functions are involved we always assume this normalization is in force. There is a natural subspace of $B$ denoted as $B_{0}$ and defined as 


$$
B_{0} \equiv\left\{f \in B: \lim _{|z|+1}\left(1-|z|^{2}\right)\left|f^{\prime}(z)\right|=0\right\}
$$

It is straightforward to check that $B_{0}$ is a closed subspace of $B$. Also if $f \in B_{0}$ and $f_{\rho}(z)=f(\rho z)$ then $f_{\rho}$ tends $f$ in $B_{0}$ as $\rho / 1$. Since each $f_{\rho}$ can be uniformly approximated on $D$ by polynomials we see that polynomials are dense in ${ }^{B_{0}}$. Thus $B_{0}$ is a separable closed subspace of B. B is nonseparable as we can easily see by checking the norms of $\left(\log \left(1-z e^{i h}\right)-\log (1-z)\right.$ for $h>0$. There is one result which is very useful and should be noted.

$$
M(f)=M(f \circ \phi)
$$

for every $\phi \in$ Aut(D). Although there is no natural inclusion relationship between the Hardy spaces $\mathrm{H}^{\mathrm{P}}$ and $\mathrm{B}\left(1 . e . \mathrm{g}(\mathrm{z})=(\log (1-z))^{2} \in \mathrm{H}^{\mathrm{P}}, \mathrm{p}^{<\infty}\right.$ yet $8 \ddagger$ B) there are some other interesting containment relations. Let us show for example that each holomorphic function with finite Dirichlet integral is in B. Assume $f(z)=\sum_{n=1}^{\infty} a_{n} z^{n}$ and

$$
\int_{D}|f(z)|^{2} \operatorname{do}(z)=\sum_{1}^{\infty} n\left|a_{n}\right|^{2}<\infty \text {. }
$$

Then for $|z|=r<1$

$$
\begin{aligned}
& \left(1-r^{2}\right)\left|f^{\prime}(z)\right| \leq\left(1-r^{2}\right)\left[\sum_{1=n}^{N} n\left|a_{n}\right| r^{n-1}\right] \\
& \left(1-r^{2}\right) \sqrt{\sum_{N+1=n}^{\infty} n\left|a_{n}\right|^{2}} \sqrt{\sum_{N+1=n}^{\infty} n r^{2 n-2}} \\
& \leq\left(1-r^{2}\right)\left[\sum_{1}^{N} n\left|a_{n}\right| r^{n-1}\right]+\left(\sum_{N+1=n}^{N} n\left|a_{n}\right|^{2}\right)^{\frac{1}{2}}
\end{aligned}
$$

For each $\epsilon>0$ we may choose $N$ so that the last square root is less than $\sqrt{\varepsilon}$. 
Hence,

$$
\lim _{r \rightarrow 1} \sup \left(1-r^{2}\right)\left|f^{\prime}(z)\right| \leq \sqrt{\varepsilon}
$$

and $f \in B_{0}$.

For a second and less obvious example we consider an $F \in L^{2}(\partial D)$. Let $I(F)$ be the average of $F$ over an interval $I \subset \partial D,|I|=$ the measure of $I$, and

$$
I(F)=\frac{1}{|I|} \int_{I} F(\theta) d \theta .
$$

$F$ is said to have bounded mean oscillation if

$$
\sup _{I \subseteq \partial D}\left[\frac{1}{|I|} \int_{I}|F(x)-I(F)| d x\right]<\infty .
$$

In the case of a function $f$ in the Hardy space $H^{2}(D)$ this is equivalent to being able to write $f$ as the sum of two holomorphic functions $f_{1}$ and $f_{2}$ for which $\operatorname{Re} f_{1}$ and $I m f_{2}$ are bounded. This is a nontrivial equivalence and the reader might wish to refer to the notes of Sarason [ 21 ] for a comprehensive discussion of this topic. But if $f$ is decomposed as above

$$
f(z)=f_{1}(z)+f_{2}(z)
$$

since $f_{1}$ and $f_{2}$ are Bloch functions it follows that $f$ is a Bloch function. Thus BMOA (the space of analytic functions with bounded mean oscillation) is a subspace of $B$. We establish now the basic duality relationships. The fundamental Idea really begins with the paper of Rubel and Shields [ 18 ]. In that 
paper the following basic principle is confirmed. Loosely stated it shows that the second dual of a Banach space of holomorphic functions satisfying a "little oh" growth condition is isomorphic to the Banach space of analytic functions satisfying the corresponding "big oh" relationship.

Theorem (3.1). The inclusion mapping of $B_{0}$ into $B$ extends to an isomorphism of $B_{0}^{* *}$ onto $B$.

We prove this theorem by a sequence of results. It is necessary to introduce an auxiliary space $I$. $I$ is the space of functions $g$ holomorphic on $D, g(0)=0$ and

$$
\int_{D}\left|g^{\prime}\left(r e^{i \theta}\right)\right| d r d \theta<\infty
$$

The functions in $I$ are the normalized holomorphic functions whose derivaties are in the Bergman one space. One checks that $I \subseteq H^{1}(D)$ and hence each $g \in I$ has well defined boundary values

$$
\lim _{r \rightarrow 1} g\left(r e^{i \theta}\right)=g\left(e^{i \theta}\right) \quad \text { a.e. }
$$

with $g\left(e^{1 \theta}\right) \in L^{\prime}(\partial D)$. The next lemma is crucial to the development.

Lemma (3.2). For $f(z)=\sum_{n=1}^{\infty} a_{n} z^{n} \in B$ and $g(z)=\sum_{n=1}^{\infty} b_{n} z^{n} \in I$ the Hadamard convolution

$$
h(z)=f * g(z)=\sum_{n=1}^{\infty} a_{n} b_{n} z^{n}
$$

is in the disc algebra. 
Proof. The function $h$ is analytic on $D$. We show that it is uniformly continuous on $D$ and so can be extended to a continuous function on $\bar{D}$. Let $z=r e^{1 \theta}$ and $\zeta \in D$,

$$
\begin{aligned}
\frac{1}{\pi} \int_{0}^{1} \int_{0}^{2 \pi}\left(1-r^{2}\right) f^{\prime}(\zeta \bar{z})(z g(z)) \cdot e^{-1 \theta} d \theta d r= \\
\sum_{n=1}^{\infty} a_{n} b_{n} \zeta^{n-1} .
\end{aligned}
$$

Also

$$
\begin{gathered}
\frac{1}{\pi} \int_{0}^{1} \int_{0}^{2 \pi}\left|g\left(r e^{1 \theta}\right)\right| d \theta d r \\
\leq \frac{1}{\pi} \int_{0}^{1} \int_{0}^{2 \pi} \int_{0}^{r}\left|g^{\prime}\left(t e^{1 \theta}\right)\right| d t d r d \theta \\
\leq\left.|| g\right|_{I} .
\end{gathered}
$$

We find using this result

$$
\begin{gathered}
|h(\zeta)| \leq c\left(\sup \left(1-|z|^{2}\right)\left|f^{\prime}(\zeta \bar{z})\right|: z \in \mathbb{D}\right)|| g||_{I} \\
\leq\left. c|| f||_{B} \cdot|| g\right|_{I} .
\end{gathered}
$$

Let points $\zeta_{1}, \zeta_{2}$ be given in $D$ and observe

$$
\left|h\left(\zeta_{1}\right)-h\left(\zeta_{2}\right)\right| \leq c|| f||_{B} \cdot\left\|g_{\zeta_{1}}-g_{\zeta_{2}}\right\| I_{I}
$$

where $g_{\zeta}(z)=g(z \zeta)$. It is routine to check that as $\zeta \rightarrow 1 \quad(|\zeta|<1)$

$$
\left\|8-g_{\zeta}\right\|_{I} \rightarrow 0
$$


Hence, $h$ is uniformly continuous on D.

Theorem (3.3). The spaces $B$ and $I^{*}$ are isomorphic. The pairing is given by $\psi \rightarrow f$ where

$$
\begin{gathered}
\psi(g)=\langle f, g\rangle \equiv \lim _{r \rightarrow 1}\left(\sum_{n=1}^{\infty} a_{n} b_{n} x^{n}\right), \\
f(z)=\sum_{1}^{\infty} a_{n} z^{n} \in B, \quad g(z)=\sum_{1}^{\infty} b_{n} z^{n} \in I .
\end{gathered}
$$

Proof. In the notation of Lemma (3.2) we have for $f \in B$ and $g \in I$

$$
|h(1)|=\left|f *_{g}(1)\right|=|\langle f, g\rangle| \leq c|| f||_{B} \cdot|| g||_{I} \text {. }
$$

Hence each $f \in B$ can be identified with a $\psi$ in $I^{*}$. Now assume $\psi \in I^{*}$ and let $a_{n}=\psi\left(z^{n}\right)$ for $n \geq 1$. The function $f(z)=\sum_{n=1}^{\infty} a_{n} z^{n}$ is analytic in $D$. Let $0<\rho<1$ and select $g \in I$, $g(z)=\sum_{1}^{\infty} b_{n} z^{n}$

$$
\psi\left(g_{p}\right)=\sum_{n=1}^{\infty} a_{n} b_{n} p^{n}=\left\langle g_{\rho}, f\right\rangle .
$$

But $g_{p}$ tends to $g$ In $I^{\cdot}$ and hence the analytic function $f$ corresponds to $\psi$. It remains to prove that $f$ is in $B$. The kernel

$$
\begin{aligned}
& g_{\zeta}(z)=z(1-\zeta z)^{-2} \text { is in } I \text { for } \zeta \text { fixed in } D \text {. With } z=r e^{1 \theta} \\
& \left\|g_{\zeta}\right\|_{I} \leq c \int_{0}^{1} \frac{1}{(1-|\zeta| r)}\left(\frac{1}{2 \pi} \int_{0}^{2 \pi} \frac{d \theta}{|1-\zeta z|^{2}}\right) d r \\
& \leq c\left(1-|\zeta|^{2}\right)^{-1} \text {. }
\end{aligned}
$$

Thus

$$
\left|f^{\prime}(\zeta)\right|=\left|<f, g_{\zeta}>\right| \leq c \cdot|| \psi|| \cdot\left(1-|\zeta|^{2}\right)^{-1}
$$


and $80 \mathrm{f}$ is in $B$.

The next theorem will be the last result we need. This result and Theorem (3.3) will immediately yield Theorem (3.1).

Theorem (3.4). The spaces $I$ and $B_{0}^{*}$ are isomorphic. The pairing is given by $\psi \rightarrow 8$, with

$$
\psi(f)=\langle f, g\rangle
$$

for each $f \in B_{0}$.

Proof. Let $\psi \in B_{0}^{*}$ and form the holomorphic function $g(z)=\sum_{n=1}^{\infty} b_{n} z^{n}$, $\psi\left(z^{n}\right)=b_{n}, n \geq 1$. For $\rho \in(0,1)$ and $f \in B_{0}$,

$$
\left|\psi\left(f_{\rho}\right)\right|=\left|\left\langle f_{\rho}, g\right\rangle\right|=\left|\left\langle f, g_{\rho}\right\rangle\right| \leq c|| \psi|| \cdot|| f|| .
$$

But for any $f \in B, f_{\rho} \in B_{0}$ hence

$$
\left\|g_{p}\right\|_{I}=\sup _{\|f\|=1}\left|\left\langle f, g_{p}\right\rangle\right| \leq c\|\psi\| \text {. }
$$

Thus $g \in I$.

We have already observed that $g$ induces a bounded linear functional on ${ }^{B}{ }_{0}$

We have already noted that for $f(z)=\sum_{n=1}^{\infty} a_{n} z^{n} \in B$ the mapping

$$
\text { m : } \mathrm{B} \rightarrow \ell^{\infty}
$$

defined by

$$
m(f)=\left(a_{n}\right)
$$


is a one to one continuous linear mapping. It can also be shown if

$\left(n_{k}\right)$ is a strictly increasing sequence of positive integers with

$n_{k+1 / n_{k}} \geq 2$, and $\left(a_{k}\right) \in l^{\infty}$ then the function

$$
f(z)=\sum_{k=1}^{\infty} a_{k} z^{n_{k}}
$$

is in $B$ and $\|f\| \leq 4\left\|\left(a_{n}\right)\right\| \infty$. Hence, the mapping

$$
\mathbf{n}: \ell^{\infty} \rightarrow \mathbf{B}
$$

given by

$$
\ell\left(a_{k}\right)=\sum_{k=1}^{\infty} a_{k} z^{2^{k}}
$$

is a (continuous) isomorphism onto its range. In particular the map $P: B \rightarrow B$ given by

$$
P\left(\sum_{1}^{\infty} a_{n} z^{n}\right)=\sum_{k=1}^{\infty} a_{2} k^{z^{2}}
$$

is a projection. Although this is not sufficient to conclude that $B$ is linearly isomorphic to $l^{\infty}$ one might anticipate this result. A denouement to the results of this nature was given by Shields and Williams [ 22 ]. Their work coupled with a result of Lindenstrauss and Pelczynski [ 16 ] will show that indeed $B$ is isomorphic to $l^{\infty}$. They also obtain the result that $B_{0}$ is isomorphic to $c_{0}$ (sequences tending to zero) with the usual sup norm (Shields and williams, [ 22,23 ]). Shields and Williams establish a direct sum decomposition for $L^{1}(D)$ in terms of a copy of and a complemented subspace. One can then apply the result of Lindenstrauss and Pelczynsk 1 on bounded projects to prove that $I$ is 1somorphic to $l$. Thus

$$
I * \simeq \ell^{\infty} \simeq B
$$


We prefer to use the notation of Shields and Williams and simply 1dentify notational connections necessary. To begin they choose positive continuous functions $\phi$ and $\psi$ on $[0,1)$ with $\phi(r) \rightarrow 0$ as $r \rightarrow 1$ and

$$
\int_{0}^{1} \psi(r) d r<\infty .
$$

The space $A(D)$ consists of holomorphic functions $f$ on $D$. Three other spaces are denoted by

$$
\begin{aligned}
& A_{\infty}=A_{\infty}(\phi) \equiv\{f \in A(D): \sup \{|f(z)| \phi(|z|): z \in \mathbb{D}\}<\infty\} \\
& A_{0}=A_{0}(\phi) \equiv\left\{f \in A(D): \lim _{|z|+1}|f(z)| \phi(|z|)=0\right\} \\
& A^{1}=A^{1}(\psi) \equiv\left\{f \in Z(D): \int_{\mathbb{D}}|f(z)| \psi(|z|) \text { do }(z)<\infty\right\}
\end{aligned}
$$

where $\sigma$ is normalized Lebesgue measure on $D$. We make the obvious identifications and isomorphic correspundences

$$
\phi(|z|)=\left(1-|z|^{2}\right), \psi(|z|) \equiv 1, \quad B \simeq A_{\infty}, \quad B_{0} \simeq A_{0}
$$

and $I \simeq \mathrm{A}^{1}$. Letting $M(D)$ denote the Banach space of complex-valued, bounded Borel measures on D (with variation norm) and $C_{0}(D)$ the Banach space of continuous functions on $\bar{D}$ such that each $f \in C_{0}$ (D) vanishes on $\partial D$ we define some isometries. For $f \in A_{\infty}, \quad g \in A^{1}$ we define

$$
T_{\infty} f=\phi f, \quad T_{1} g=\psi g \text { and } M g=g \psi d \sigma
$$

Then $T_{\infty}$ is an isometry of $A_{\infty}$ to $L^{\infty}(D)\left(\left.T_{\infty}\right|_{A_{0}} \equiv A_{0}\right.$ is an isometry of $A_{0}$ to $\left.C_{0}\right)$ and 
$T_{1}$ is an isometry of $A^{1}$ to $L^{1}$ and $M$ is an isometry to $M(D)$. The following nötational pairing is used

$$
\begin{aligned}
(f, g) & =\int_{D} f(z) g(\bar{z}) \phi(|z|) \psi(|z|) d \sigma(z) \\
& =\int_{D} f(z) g(\bar{z})\left(1-|z|^{2}\right) d \sigma(z)
\end{aligned}
$$

with $f \in A_{\infty}, g \in A^{1}$. For example if $f$ and $g$ are polynomials

$$
\begin{gathered}
f(z)=\sum a_{n} z^{n} \quad \text { and } \quad \dot{g}(z)=\sum b_{n} z^{n} \\
(f, g)=\sum \frac{a_{n} b_{n}}{(n+1)(n+2)} .
\end{gathered}
$$

The pair $\{\phi, \psi\} \quad$ is a normal pair with $\varepsilon=\frac{1}{2}, k=\frac{3}{2}$ and $\alpha=1$.

Lemma (3.5). Let $z$, w $\in D$

$$
K_{w}(z) \equiv 2(1-w z)^{-(3)}
$$

Then
(1) $g(w)=\left(K_{w}, g\right)$ all $g \in A^{1}$;
(2) $f(w)=\left(f, K_{w}\right)$ all $f \in A_{\infty}$;
(3) $\operatorname{span}\left\{K_{w}: w \in D\right\} \quad$ is dense in $A^{1}$ and $A_{0}$;
(4) span $\left\{K_{w} ; w \in D\right\} \quad$ is weak star dense in $A_{\infty}$.

Proof. This is just a computation and we limit ourselves to proving the case of $A^{1}$ in (3) and part (4). By the Hahn-Banach theorem it suffices to show that if $h \in L^{\infty}(D)$ and if

$$
\int_{D} K_{w}(z) h(\bar{z}) d \sigma(z)=0
$$


for all $w \in D$, then $h$ amihilates all of $T_{1} A^{1}=T A^{1}$. A calculation yields

$$
0=2 \sum_{n=0}^{\infty}(n+1)(n+2) w^{n}\left(\int_{0} z^{n} h(\bar{z}) d \sigma(z)\right)
$$

for all $w \in D$. Hence, $h$ annihilates all polynomials. The polynomials are dense in $A^{1}$ and thus span $\left\{K_{w}\right\}$ is dense in $A^{1}$.

For part (4) we recall that if $\sigma_{n}(f)$ denotes the $n t h$ Cesaro means of the partial sums of the Taylor series for $f$ then

$$
\sup _{0 \leq \theta<2 \pi}\left|\sigma_{n}(f)\left(r e^{1 \theta}\right)\right| \leq \sup _{0 \leq \theta<2 \pi}\left|f\left(r e^{1 \theta}\right)\right| .
$$

Hence,

$$
\begin{aligned}
\sup _{0 \leq r<1} & {\left[\sup _{\theta}\left|\sigma_{n}(f)\left(r e^{1 \theta}\right)\right|\left(1-r^{2}\right)\right] } \\
& \leq \sup _{0 \leq r<1}\left[\left(\sup \left|f\left(r e^{1 \theta}\right)\right|\right)\left(1-r^{2}\right)\right] .
\end{aligned}
$$

If $f \in A_{\infty}$ we apply the above inequality to conclude that $\left(1-r^{2}\right) \sigma_{n}(f)\left(r e^{i \theta}\right)$ converges pointwise and boundedly to $\left(1-r^{2}\right) f\left(r e^{i \theta}\right)$. By the Lebesgue dompinated convergence theorem we have

$$
\begin{aligned}
\lim _{n} & \int_{D}\left(1-|z|^{2}\right) \sigma_{n}(f)(z) h(\bar{z}) d \sigma(z) \\
& =\int_{D}\left(1-|z|^{2}\right) f(z) h(\bar{z}) d \sigma(z)
\end{aligned}
$$

for all $h \in L^{\prime}(D)$. Thus each $f$ in $A_{\infty}$ is the weak star limit of a sequence of polynomials. 
The $L^{1}(D)-L^{\infty}(D)$ duality implies that each weak star continuous functional on $A_{\infty}$ is given by

$$
\int\left(1-|z|^{2}\right) f(z) h(\bar{z}) d \sigma(z)
$$

where $h \in L^{1}(D)$. But as above if

$$
\int_{0} K_{w}(z) h(\bar{z})\left(1-|z|^{2}\right) d \sigma(z)=0
$$

then $h$ annihilates polynomials and thus span $\left\{K_{w}\right\}$ is weak star dense in $\mathrm{A}_{\infty} \cdot$

The next lemma is well known and the second follows by a calculation.

Lemma [3.6]. For $0<\mathbf{r}<1$,

$$
\int_{0}^{2 \pi}\left|1-r e^{1 t}\right|^{-2} d t=0\left((1-r)^{-1}\right)
$$

Lemma [3.7]. For $m \geq 2$ and $0 \leq \rho<1$

$$
\int_{0}^{1}(1-\rho r)^{-m} d r=0\left(\left(1-\rho^{2}\right)^{1-m}\right)
$$

Theorem 3.8. The transformation $Q$ defined by

$$
(2) \mu(w)=\int K_{w}(\bar{z})\left(1-|z|^{2}\right) d_{\mu}(z)
$$

with $\mu \in M(D), w \in \mathbb{D} \quad$ is a bounded operator mapping $M(D)$ onto $A^{1}$.

The transformation $2_{1} \equiv 2 L_{L^{1}(D)}$ is a bounded operator mapping $L^{1}$ (D) onto $A^{1}$. The operator $T_{1} Q_{1}$ is a bounded projection of $L^{1}(D)$ onto the subspace $\mathrm{TA}^{1}$.

Proof. We consider only $Q_{1}$ the proof for 2 is analogous. For 
400 J. A. CIMA

$f \in L^{1}(D)$

$$
\begin{gathered}
\int_{\mathbb{D}}\left|\left(21^{f}\right)(w)\right| d \sigma(w) \leq \\
\int_{\mathbb{D}}\left(\int_{\mathbb{D}}\left|K_{w}(\bar{z})\right| d \sigma(w)\right)\left(1-|z|^{2}\right)|f(z)| d \sigma(z) \\
\leq c \cdot \int_{\mathbb{D}}|f(z)| d \sigma(z),
\end{gathered}
$$

where we have used Lemmas 3.6 and 3.7 . If $Q_{1} f=g$,

$$
\left(Q_{1}^{2} f\right)(w)=\left(K_{w}, g\right)=g(w)=\left(Q_{1} f\right)(w) .
$$

Hence, $Q_{1}$ is a bounded projection from $L^{1}(D)$ onto $A^{1}$.

Corollary 3.9. We have the following direct sum decomposition

$$
\begin{gathered}
L^{1}(D)=T_{1} A^{1} \bullet\left(T_{\infty} A_{\infty}\right)^{\perp} \\
\left(T_{\infty} A_{\infty}\right)^{\perp}=\left\{g \in L^{\prime}(D):(\phi f, g)=0\right. \\
\text { all } f \in A\}
\end{gathered}
$$

Proof. We show that the null space of $Q_{1}\left(=T_{1} Q_{1}\right)$ is $\left(T_{\infty} A_{\infty}\right)^{\perp}$. A $g \in \mathrm{L}^{1}(D)$ is in the kernel of $Q_{1}$ if and only if

$$
0=\int_{D}\left(1-|z|^{2}\right) K_{w}(\bar{z}) g(z) d o(z)
$$

for all $w \in D$. The finite linear comblnations of $K_{w}$ are weak star dense in $A_{\infty}$ (which is weak star closed) and hence $g \in\left(T_{\infty} A_{\infty}\right)^{\perp}$.

Theorem 3.10. $\mathrm{A}^{1}$ is topologically isomorphic to $\ell^{1}$. 
Proof. Let $\left\{r_{n}\right\}$ be a strictly increasing sequence of positive numbers with $\lim r_{n}=1$. Let $A_{j}$ denote the annulus

$$
A_{j}=\left\{z \in D: z_{j-1} \leq|z|<r_{j}\right\}
$$

$1 \leq j$. Let $R_{j}: L_{1}(\mathbb{D}) \rightarrow L_{1}\left(A_{j}\right), j=1,2, \ldots$ be the natural projection (restriction), with respect to Lebesgue measurə. The operator

$$
R: L_{1}(D) \rightarrow\left(L_{1}\left(A_{1}\right) \oplus L_{1}\left(A_{2}\right) \oplus L_{1}\left(A_{3}\right) \oplus \cdots\right.
$$

defined by

$$
R f=\left(R_{1} f, R_{2} f, \cdots\right)
$$

is an onto isometry.

A normal families argument shows that $\left.R_{j}\right|_{A^{1}}$ is a compact operator. Hence, if $S$ is the unit ball in $A^{1}$, then $R_{j} S$ is a totally bounded set in $L_{1}\left(A_{j}\right)$.

Consider $E \subseteq \mathbb{R}^{2}$ a Lebesgue measurable set and do Lebesgue measure on E... Further assume with out loss of generality that $\sigma(E)=1$. For each $n$, let $P_{n}$ be a partition of $E$ into disjoint measurable sets $E_{1}^{n}, \ldots, E_{2^{n}}^{n}$ with $\mu\left(E_{1}^{n}\right)=\frac{1}{2^{n}}$ for each $i$. Assume that $P_{n+1}$ refines $P_{n}$ for each $n$. Now for $f \in L_{1}(E, \mu)$, define

$$
P_{n} f=\sum_{i=1}^{2^{n}} \frac{1}{\mu\left(E_{i}^{n}\right)}\left(\int_{E_{i}^{n}} f d\right) x_{E_{i}^{n}}
$$

Of course $X_{E_{1}^{n}}$ is the characteristic of the set $E_{i}^{n}$. One can prove that $P_{n} f \rightarrow f$ in $L_{1}$ norm, uniformly on compact subsets of $L_{1}(E, \mu)$. Each $P_{n} f$ is of the form

$$
\sum_{i=1}^{2^{n}} c_{i} x_{E_{i}^{n}}=P_{n} f .
$$


Hence the map

$$
\sum_{i=1} C_{1} x_{E_{1}^{n}} \rightarrow\left(\frac{C_{1}}{2^{n}}, \cdots, \frac{c_{n}}{2^{n}}\right)
$$

is an isometry of the range of $P_{n}$ onto $\ell_{1}^{2^{n}}$. One can check that $P_{n}^{2}=P_{n}$ and hence $P_{n}$ is a projection.

There is for every given $\varepsilon_{j}>0$ a projection $P_{j}=P_{j}\left(\varepsilon_{j}\right)$ of $L_{1}\left(A_{j}\right)$ into itself is given so that $\left\|P_{j}\right\| \leq 1, P_{j} L_{1}\left(A_{j}\right)$ is isometric to some $\ell_{1} k_{j}$ for a suitable integer $k_{j}$ and

$$
\| P_{j} R_{j} f-R_{j} f|| \leq \varepsilon_{j}
$$

for every $f \in S$. It is now clear that the subspace $X$,

$$
X=\left(P_{1} L_{1}\left(A_{1}\right) \oplus P_{2} L_{1}\left(A_{2}\right) \oplus \ldots\right)
$$

of

$$
\mathrm{L}_{1}(D)=\mathrm{L}_{1}\left(\mathrm{~A}_{1}\right) \oplus \mathrm{L}_{1}\left(\mathrm{~A}_{2}\right) \oplus \cdots
$$

is isometric to $\ell_{1}$.

The operator $T: R A_{1} \rightarrow X$ defined by

$$
T\left(R_{1} f, R_{2} f, \ldots\right)=\left(P_{1} R_{1} f, P_{2} R_{2} f, \ldots\right)
$$

satisfies

$$
\|T R f=R f\| \leq \sum_{j=1}^{\infty}\left\|P_{j} R_{j} f-R_{j} f\right\| \leq\left(\sum_{1}^{\infty} \varepsilon_{j}\right)
$$

for $f \in S$. Thus, if $\varepsilon>0$ is given we can choose the $\varepsilon_{j}>0$ so that $\left(\sum_{1}^{\infty} \epsilon_{j}\right)<\epsilon$ or

$$
|| T R f-R f||<\varepsilon \| R f||
$$

for $f \in A_{1}$. 
Since $\mathrm{RA}_{1}$ is complemented in $\mathrm{R} \mathrm{L}_{1}$ we claim that for $\epsilon>0$ sufficiently small $T R A_{1}$ is complemented in $R_{1}$ and so in $X$. Let us consider the general situation of a Banach space $U$ with $Y$ a complemented subspace of $U$. Then $U=Y \oplus Z$ and there is a projection $P$ on $U$ with range $P$ equal $Y$. We claim there is $\frac{1}{2}>n>0$ such that if $\|y\|=1, \quad y \in Y$ then

$$
(|| x-y||<2 \eta) \cap z=\phi
$$

For if not $y_{n} \in Y, z_{n} \in Z$ with

$$
|| y_{n}-z_{n}||<\frac{1}{n} .
$$

Thus

$$
|| \mathrm{P}|| / \mathrm{n} \geq|| \mathrm{P}\left(\mathrm{y}_{\mathrm{n}}-\mathrm{z}_{\mathrm{n}}\right)||=1
$$

and this is absurd. Now let $T$ be an operator on $Y$ into $U$ which satisfies

$$
\|\mathrm{Ty}-\mathrm{y}\| \leq n|| \mathrm{y} \|
$$

We claim that $T(Y)$ is a complemented subspace of $U$. First, $T$ is invertible as an operator on $Y \rightarrow T(Y)$. Next define $\tilde{T}$ on all of $U$ as follows,

$$
\begin{aligned}
& \tilde{T}(y)=T(y), y \in Y \\
& \tilde{T}(z)=z, z \in Z .
\end{aligned}
$$

Computations show $\tilde{T}$ is linear on $U$ and is one to one. For if there is a $\mathrm{y}_{0} \in \mathrm{Y},\left\|\mathrm{y}_{0}\right\|=1$ and $\mathrm{Ty}_{0}=\mathrm{z}_{0} \in \mathrm{z}$, then 
404

$$
\text { J. A. CIMA }
$$

$$
2 n<|| \mathrm{Ty}_{0}-\mathrm{y}_{0} \|=|| \mathrm{z}_{0}-\mathrm{y}_{0}||<n
$$

Next if ||$y \oplus z|| \leq 1$ then

$$
\|z|| \leq|| y \oplus z||+|| P(y \oplus z)|| \leq 1+\| P \| \leq M \text {. }
$$

Similarly, ||$y|| \leq M$ if ||$y \oplus z||=1$. Hence,

$$
|| \tilde{T}(y \oplus z)|| \leq|| T|||| y||+|| z|| \leq M
$$

This establishes the claim that $T R A_{1}$ is complemented in $R L_{1}$. But it is known that an infinite-dimensional complemented subspace of $\ell_{1}$ is isomorphic to $\ell_{1}$ and since $T$ is an isomorphism we find that $A_{1}$ is isomorphic to $\ell_{1}$.

There are other results of interest which are known in regard to the Banach space structure. Anderson and Shields [ 5 ] have characterized the multipliers from $B$ into $\ell^{P}$ in the following sense. If $A$ and $B$ are two vector spaces of sequences the multipliers from $A$ to $B$, denoted $(A, B)$ are defined as

$$
\begin{aligned}
(A, B)= & \left\{\lambda=\left\{\lambda_{n}\right\}:\left\{\lambda_{n} a_{n}\right\} \in B\right. \text { for } \\
& \text { every } \left.\left\{a_{n}\right\} \in A\right\} .
\end{aligned}
$$

For $I_{n}=\left\{k: 2^{n} \leq k<2^{n+1}\right\}, n=0,1,2, \ldots$ and $1 \leq \alpha, \beta \leq \infty$ denote by $\ell(\alpha, \beta)$ the set of sequences $\left\{a_{k}\right\} \quad(k \geq 1)$ for which

$$
\left\{\left(\sum_{I_{n}}\left|a_{k}\right|^{\alpha}\right)^{\frac{1}{2}}\right\}_{n=0}^{\infty} \in e^{\beta} \quad(\alpha<\infty)
$$

and

$$
\left\{\sup _{k \in I_{n}}\left|a_{k}\right|\right\}_{n=0}^{\infty} \in e^{\beta} \quad(\alpha=\infty)
$$


These are Banach spaces when they are given the obvious norm. Anderson and Shields prove that

$$
\left(B, l^{p}\right)=\left\{\begin{array}{ll}
\ell\left(\frac{2 p}{2-p}, p\right) & 1 \leq p \leq 2 \\
\ell(\infty, p), & 2 \leq p \leq \infty
\end{array} .\right.
$$

Questions of rotundity of the unit ball have also been investigated. Examples of extreme points in the unit ball of ${ }_{0}$ are $\alpha_{n}{ }^{n}, n \geq 2$, and other powers of Blaschke factors, suitably normed e.g.

$$
\alpha_{n}\left[\left(\frac{z-\alpha}{1-\bar{\alpha}^{2}}\right)^{\mathrm{n}}-(-\alpha)^{\mathrm{n}}\right], \quad|\alpha|<1
$$

No other examples of extreme points are known. A characterization of the extreme points of the unit ball of $B_{0}$ is given in Cima and Wogen [ 8 ]. We are considering functions $f$ in ${ }^{B}{ }_{0}$ with $\|f\|=1$. Let

$$
L_{f}=\left\{z \in D:\left|f^{\prime}(z)\right|\left(1-|z|^{2}\right)=1\right\}
$$

Theorem 3.11. Let $f$ be in the unit ball of $B_{0}$. Then $f$ is an extreme point If and only $L_{f}$ is an infinite set.

A second result is also noteworthy in this regard.

Theorem 3.12. If $f$ is an extreme point of the unit ball of $B_{0}$ then there are simple closed pairwise disjoint analytic curves $\gamma_{1}, \gamma_{2}, \ldots, \gamma_{k}$ with $k \geq 1$ and points $w_{1}, w_{2}, \ldots, w_{j}$ with $j \geq 0$ so that

$$
L_{f}=\left(\bigcup_{i=1}^{k} \gamma_{i}\right) \cup\left\{w_{1}, w_{2}, \ldots, w_{j}\right\} \text {. }
$$


There are other examples of extreme points of the unit ball in $B$. The function $\log \left(\frac{1+z}{1-z}\right)$ is an example and scalar multiplies of singular inner functions corresponding to a point mass on $\partial D$ are also examples.

The observation that

$$
\begin{gathered}
M(f)=\sup \left\{\left|f^{\prime}(z)\right|\left(1-|z|^{2}\right): z \in \mathbb{D}\right\} \\
=M(f \circ \phi)
\end{gathered}
$$

for every a $\in$ Aut(D) leads one to consider the isometries of $B_{0}$ and $B$. In particular the extreme points of the unit ball are permuted under an onto isometry. The process of composing a $\phi \in$ Aut(D) with $f \in B$ or $B_{0}$ is a composition operator on the space. We write

$$
\mathrm{C}_{\phi}: \mathrm{B}_{0} \rightarrow \mathrm{B}_{0} \quad(\text { or } \mathrm{B} \rightarrow \mathrm{B})
$$

and this is defined as

$$
C_{\phi}(f)(z)=f \circ \phi(z)
$$

In [ 9 ] Cima and Wogen have proven the following results.

Theorem 3.13. If $\mathrm{S}: \mathrm{B}_{0} \rightarrow \mathrm{B}_{0}$ is an isometry, then

$$
\text { (Sf) }(z)=\lambda f(\phi(z))-\lambda f(\phi(0)) \text {, }
$$

where $\lambda \in \partial \mathbb{D}$ and $\phi \in$ Aut (D).

Corollary 3.14. Every isometry of $B_{0}$ is onto.

Theorem 3.15. If $S: B \rightarrow B$ is an onto isometry, then there is a holomorphic automorphism $\phi$ and $a \lambda \epsilon$ d such that

$$
\text { (Sf) (z) }=\lambda(f(\phi(z)))-\lambda f(\phi(0)) \text {. }
$$


We conclude with the following interesting result of Rubel and Timoney

[ 19 ]. A linear space $\mathrm{X}$ of analytic functions is said to be Mobiusinvariant if $\forall \mathrm{f} \in \mathrm{X}$ and every Mobius transformation $\phi, f \circ \phi \in \mathrm{X}$. A real valued function $\mathrm{p}: \mathrm{X} \rightarrow[0, \infty)$ is said to be a Mobius-invariant semi-norm on $X$ if $p(f \circ \phi)=p(f)$ for every Mobius transformation $\phi$ of $\mathbb{D}$ and every $f \in X$. A non-zero linear functional $L$ on $X$ is said to be decent if

$$
|L(f)| \leq M \sup \{|f(z)|: z \in K\}
$$

for all $f \in X$, for some $M>0$ and same compact subset $K$ of $\mathbb{D}$. Theorem [3.16]. Let $\mathrm{X}$ be a Mobius-invariant linear space of analytic functions on the unit disc and let $p$ be a Mobius-invariant seminorm on $\mathrm{X}$. If there exists a decent linear functional $\mathrm{L}$ on $\mathrm{X}$ continuous with respect to $\mathrm{p}$, then $\mathrm{X} \subseteq \mathrm{B}$ and there exists a constant $\mathrm{A}>0$ such that

$$
p_{B}(f) \leq A p(f)
$$

for all $f \in X$. In this theorem $P_{B}(f)$ is the seminorm given by $\mathrm{p}_{B}(f) \equiv \operatorname{Sup}_{\mathbf{z} \in \mathbb{D}}\left(1-|z|^{2}\right)\left|f^{\prime}(z)\right|$ so that $p_{B}(c)=0 \forall c \in \phi$. We observe that

$$
p_{B}(f)=\operatorname{Sup}\left\{\left|(f \circ \Phi)^{\prime}(0)\right|: \phi \in \operatorname{Aut}(\mathbb{D})\right\} .
$$

This motivates the following lemma.

Lemma [3.17]. For each $\mathrm{n} \leq 1$, the expression

$$
P_{n}(f) \equiv \operatorname{Sup}\left\{\left|(f \circ \Phi)^{n}(0)\right|: \phi \in \operatorname{Aut}(\mathbb{D})\right\}
$$


defined on analytic functions, is a seminorm equivalent to the Bloch seminorm.

Proof. We follow Rubel and Timoney and consider the case $n=3$. The general case requires more bookkeeping but is similar. Let

$\phi_{-a}(z)=(z+a)(1+\bar{a} z)^{-1}$ and observe that

$$
\left(f \circ \phi_{-a}\right)^{(3)}(0)=f^{(3)}(a)\left(1-|z|^{2}\right)-6 f^{\prime \prime}(a)\left(1-|a|^{2}\right)^{2} a+
$$

$6 f^{\prime}(a)\left(1-|z|^{2}\right) a^{-2}$. Choose $a=\left(\frac{1}{2}\right) e^{i \theta}$ and observe that

$$
\begin{aligned}
\frac{1}{2 \pi} \int_{\theta=0}^{2 \pi} a^{2}\left(f \circ \phi_{-a}\right)(3)(0) d \theta & =\frac{9}{32}\left(\frac{1}{2 \pi} \int_{0}^{2 \pi} f^{\prime}(a) d \theta\right) \\
& =\frac{9}{32} f^{\prime}(0) .
\end{aligned}
$$

Taking absolute values we find

$$
\left|f^{\prime}(0)\right| \leq\left(\frac{8}{9}\right) p_{3}(f)
$$

If $\phi$ is in Aut(D) we apply this last inequality to obtain

$$
\operatorname{Sup}\left\{\mid(f \circ \phi)^{\prime}(0): \phi \in \operatorname{Aut}(D)\right\} \leq\left(\frac{8}{9}\right) p_{3}(f)
$$

Hence, $p_{B}(f) \leq p_{3}(f)$.

For the converse we apply the Cauchy integral theorem

$$
f^{\prime \prime}(z)=\frac{1}{2 \pi} \int_{0}^{2 \pi} \frac{f^{\prime}(\zeta)}{\left(\zeta^{-z}\right)^{2}} d \theta
$$

with $|\zeta|=\frac{|z|+1}{2}$. An easy computation shows that if $\phi(0)=z$

$$
\begin{aligned}
|(f \circ \phi) "(0)| \leq c p_{B}(f)+\left|f^{\prime \prime}(z)\right|\left(1-|z|^{2}\right)^{2} \\
\quad \leq c\left(p_{B}(f)+\sup \left|f^{\prime}(\zeta)\right|\left(1-|\zeta|^{2}\right)\right) \\
\quad \leq c p_{B}(f) .
\end{aligned}
$$


A simflar estimate will show that $f^{\prime \prime \prime}(z)$ also satisfies such an inequality. Hence, there is a constant $c$ such that

$$
p_{3}(f) \leq c p_{B}(f) \text {. }
$$

Proof of the Theorem.

Let $(\mathrm{X}, \mathrm{p})$ satisfy the hypothesis of the theorem and let $\mathrm{L}$ be a linear functional satisfying

$$
L(f) \leq p(f)
$$

and

$$
|L(f)| \leq A \operatorname{Sup}\{|f(z)|: z \in K \subset \mathbb{D}\}
$$

for all $f \in X, A>0$.

By the Hahn-Banach Theorem L extends to a continuous linear functional (denoted again as $L$ ) on $H(D)$ (all holomorphic functions on $D$ ) and the inequality for $L$ on $K$ remains valid. It is well known that $L$ can be identified with a function $g(z)=\sum_{n=N \geq 0}^{\infty} b_{n} z^{-n}$ $\left(b_{N} \neq 0\right)$ holomorphic in $|z| \geq r, r<1$, in the following way

$$
L(f)=\frac{1}{2 \pi i} \int_{|z|=r} f(z) g(z) \frac{d z}{z} .
$$

Let $\phi(z)=e^{i \lambda} z$ be a rotation and change variables to deduce

$$
|L(f \circ \phi)|=\left|\frac{1}{2 \Pi I} \int_{|z|=r} f(z) g\left(e^{i \lambda} z\right) \frac{d z}{z}\right| \leq p \text { (f) }
$$

An application of Fubini's theorem produces the following equality 


$$
\frac{1}{2 \pi} \int_{\lambda=0}^{2 \pi} e^{i N \lambda} \quad\left[\frac{1}{2 \pi I} \int_{|z|=r} f(z) g\left(e^{i \lambda} z\right) \frac{d z}{z}\right]=b_{N} f^{N}(0) .
$$

This implies

$$
p(f) \geq\left|b_{N}\right|\left|f^{N}(0)\right|
$$

and replacing $f$ by $f \circ \phi$ and taking the supremum we obtain

$$
\mathrm{p}_{N}(f)=\operatorname{Sup}\left\{\left|(f \circ \phi)^{N}(0)\right|: \phi \in \operatorname{Aut}(\mathbb{D})\right\} \leq \frac{1}{\mid b_{N} T} p(f) .
$$

If $N>0$ we apply the lemma (3.17) to produce the result. If $N=0$ we conclude $\mathrm{X} \subset \mathrm{H}^{\infty}$ and that $\mathrm{p}$ dominates the supremum norm \|\|$_{\infty}$. The sup norm dominates $\mathrm{p}_{B}$ and $\mathrm{H}^{\infty} \subset \mathrm{B}$. This conclude the proof of the theorem.

4. Open questions.

Of course the older question of getting a sharp estimate for $b$ is viable. Let us define subspaces of $B$ as follows

$$
L=\{f \in B: f \text { has radial limits a.e. on } \partial D\}
$$

and

$$
M=\left\{f(z)=\sum_{1=n}^{\infty} a_{n} z^{n} \in B \quad \lim a_{n}=0\right\} .
$$

We know that if $f \in L$ then the Taylor coefficients of $f$ tend to zero (Theorem 2.8). Hence, $L \subset M$. But $M$ is closed in $B$. For if $\left\{f_{k}\right\} \subset M$ is a Cauchy sequence and $f_{k} \Rightarrow f$ in $B$ we recall that

$$
\left|a_{n}(f)-a_{n}\left(f_{k}\right)\right| \leq c|| f-f_{k}||_{B} \text {. }
$$


Hence,

$$
\left|a_{n}(f)\right| \leq c|| f-f_{k}||_{B}+\left|a_{n}\left(f_{k}\right)\right|
$$

and $\lim a_{n}(f)=0$. The following inclusions have been pointed out

$$
\mathrm{H}^{\infty} \subseteq \mathrm{BMOA} \subseteq \mathrm{B} \cap\left(\stackrel{1}{1}^{\mathrm{p}}\right) \subseteq \mathrm{M} \subseteq \mathrm{L} .
$$

It would be a useful result to describe the Bloch closure of $H^{\infty}$ or BMOA in L. A question raised by D. Campbell and the author is the following. Produce a function $f \in B \cap\left(\bigcap_{1}^{\infty} \mathrm{H}^{\mathrm{P}}\right)$ which is not in BMOA. In answer to a question posed by the author $R$. Timoney has produced a function $f \in B$ such that $f$ has radial limit values in $L^{\infty}(\partial D)$ but $f \notin \cup_{\mathrm{H}}^{\mathrm{P}}$. Are there any natural conditions which can be placed on a Bloch function $f$ so that if $f$ has radial limits in ${ }_{L^{P}}(\partial D)$ then $f \in \mathrm{H}^{\mathrm{P}}$ ?

Several questions remain concerning the structure of the unit ball in $B_{0}$. Namely, is each extreme point of the unit ball of $B_{0}$ of the form (3.1)? What is the closed convex hull of the set of functions given by (3.1)?

As a final question, we consider an oral communication of D. Sarason. There are singular functions $S *(z)$ in $B_{0}$. This is not an obvious result but follows by some work of $\mathrm{H}$. Shapiro and an application of the Zgymund criteria for $B_{0}$. Hence, for $\alpha \in D \backslash K$, where $K$ has capacity zero, the functions

$$
B_{\alpha}(z) \equiv \frac{S *(z)-\alpha}{1-\bar{\alpha} S *(z)}
$$

are $B$ laschke products in $B_{0}$ (Frostman). Give an explicit construction for $B_{\alpha}$ by designating its zeros. 


\section{REFERENCES}

1. L. V. Ahlfors, "An extension of Schwarz's lemma", Trans. Amer. Math. Soc. 43 (1938), 359-364.

2. and H. Grunsky, "Über die Blochsche Konstante", Math. Z., 42 (1937), 671-673.

3. Anderson, J. M., Clunie, J., and Pommerenke, Ch., "On Bloch functions and normal functions", J. reine angew. Math. 270, 12-37 (1974).

4. and Rubel, L. A., "Hypernormal meromorphic functions", to appear.

5. and Shields, A. L., "Coefficient multipliers of Bloch functions", Trans. Amer. Math. Soc. 224 (1976), 255-265.

6. Bloch, A., "Les theoremes de M. Valiron sur les fonctions entieres et la theorie de 1'uniformisation", Annales de la Fac. des Sc. de Toulouse, $3^{\mathrm{e}}$ Serie. 17 (1925), 1-22.

7. Bochner, S., "Bloch's theorem for real variables", Bull. Amer. Math. Soc. 52 (1946), 715-719.

8. Cima, J. A. and Wogen, W. R., "Extreme points of the unit ball of the Bloch space Bo", Mich. Math. J. 25 (1978), 213-222.

9. and , "On isometries of the Bloch space", to appear Illinois J. of Math.

10. Duren, P., "H $\mathrm{H}^{\mathrm{P}}$ Spaces", Academic Press, New York (1970).

11. , H. S. Shapiro and A. L. Shields, "Singular measures and domains not of Smirnov type", Duke Math. J., 33 (1966), 247-254.

12. Harris, L. A., "On the size of balls covered by analytic transformations", Monatsh. Math. 83 (1977), No. 1, 9-23.

13. Heins, M., "Selected topics in the classical theory of functions of a complex variable", Holt, Rinehart and Winston, New York, 1962.

14. "A class of conformal metrics", Bull. Amer. Math. Soc., 67 (1961), 475-478.

15. Lehto, 0. and Virtanen, K.J., "Boundary behaviour and normal meromorphic functions", Acta Math., 97 (1957), 47-65.

16. Lindenstrauss, J. and A. Pelczynsk1, "Contributions to the theory of the classical Banach spaces", J. Funct. Anal. 8 (1971), 225-249. 
17. Pommerenke, Ch., "On Bloch functions", J. London Math. Soc. 2 689-695 (1970).

18. Rubel, L. A. and A. L. Shields, "The second duals of certain spaces of analytic functions", J. Austral. Math. Soc. 11 (1970), 276-280.

19. and R. M. Timoney, "An extremal property of the Bloch space", to appear.

20. Sakaguchi, K., "On Bloch's theorem for șeveral complex variables", Sci. Rep. Tokyo Kyoiku Daigaku. Sect. A. 5(1956), 149-154.

21. Sarason, D., "Function theory on the unit circle", Lecture Notes, V.P.I. and S. U., Blacksburg, Va., June, 1978.

22. Shields, A. L. and D. L. Williams, "Bounded projections, duality and multipliers in spaces of analytic functions", Trans. Amer. Math. Soc. 162 (1971), 287-302.

23. and , "Bounded projections, duality and multipliers in spaces of harmonic functions", J. Reine Angew. Math., 299/300 (1978), pp. 256-279.

24. Seide1, W. and J. L. Walsh, "On the derivatives of functions analytic in the unit circle and their radii of univalence and p-valence", Trans. Amer. Math. Soc., 52 (1942), 128-216.

25. Takahashi, S., "Univalent mappings in several complex variables", Ann. of Math., 53 (1951), 464-471.

26. Zygmund, A. "Smooth functions," Duke Math. J., 12 (1945), 47-76.

\section{ERRATA}

On pages 15 and 16 :

On page 15, line $3(b)$

On page 16, 1ines 3, 4:

On page 16, line 4:

On page 33, line 6 :

$$
\begin{aligned}
& \text { Replace } f \text { by } g \\
& \text { Replace } g \text { by } s \\
& \text { Replace } g \text { by } s \\
& \text { Replace } A^{2} f\left(e^{i \theta}\right) \text { by } \\
& A^{2}\left[f\left(e^{i \theta}\right)-f\left(t e^{i \theta}\right)\right]
\end{aligned}
$$

The direct sum is taken in the $\ell^{1}$ norm. 


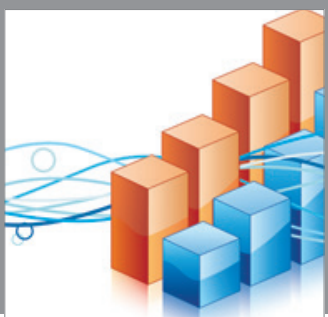

Advances in

Operations Research

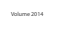

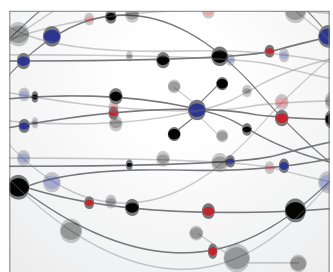

\section{The Scientific} World Journal
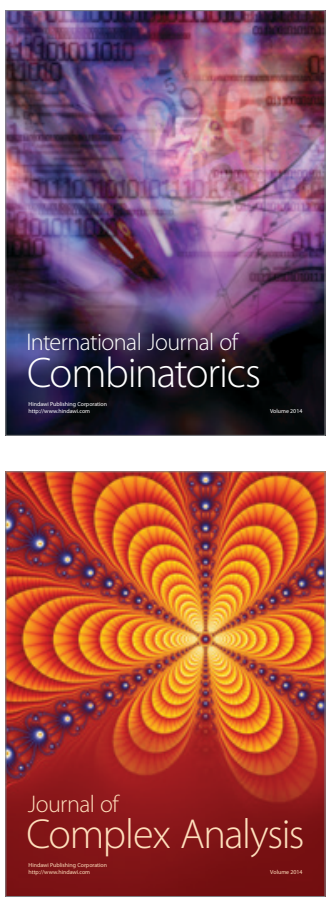

International Journal of

Mathematics and

Mathematical

Sciences
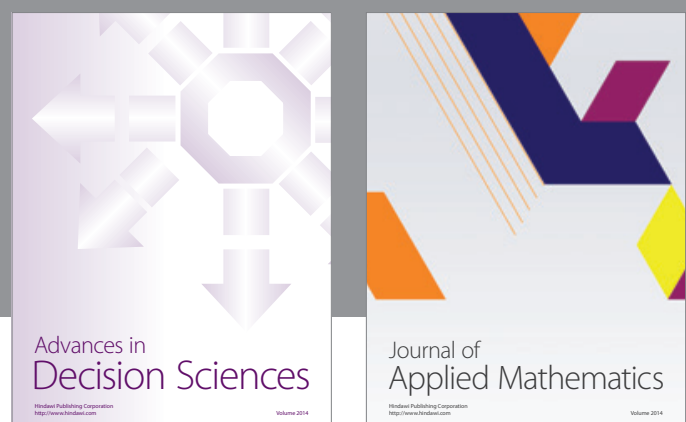

Journal of

Applied Mathematics
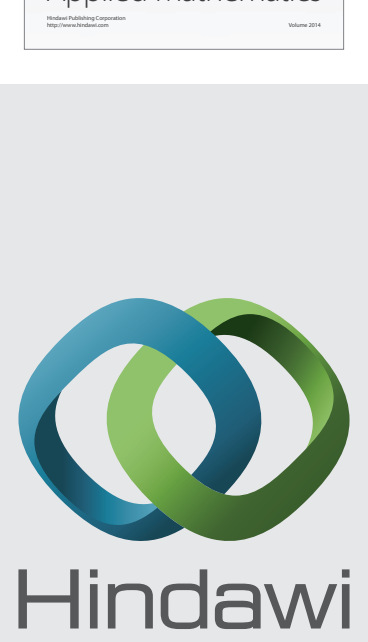

Submit your manuscripts at http://www.hindawi.com
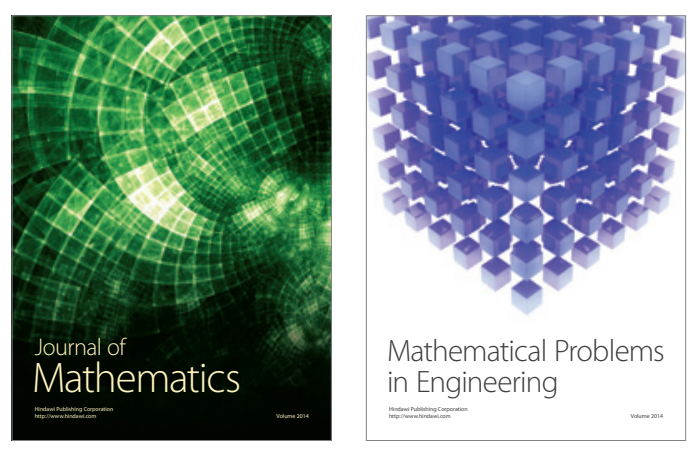

Mathematical Problems in Engineering
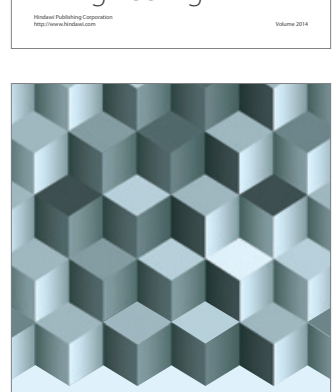

Journal of

Function Spaces
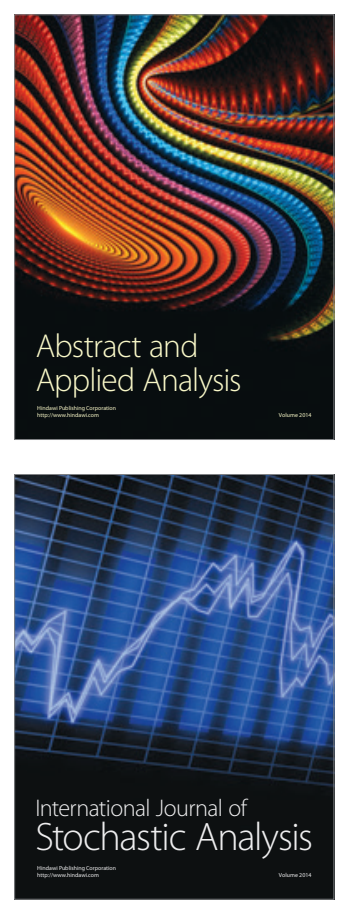

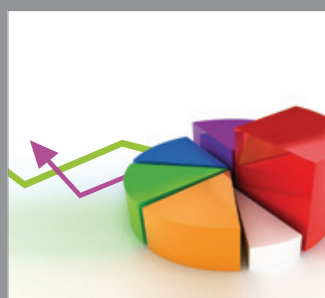

ournal of

Probability and Statistics

Promensencen
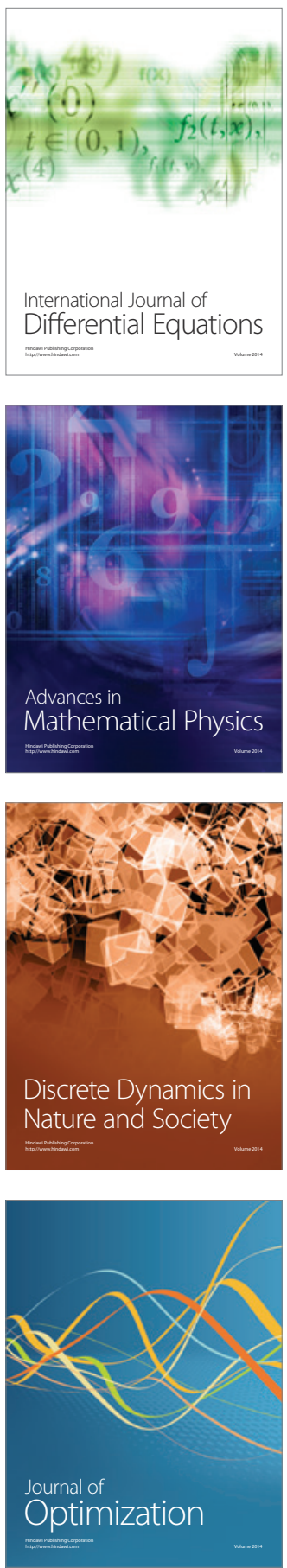